\title{
EDITORIAL
}

\section{Focus issue on biomarkers}

$\mathrm{R}$ esearch advances and the introduction of new targeted therapies for the treatment of cancer in the past decade have led to improved outcomes. As a result of these rapid changes, it has become paramount to identify prognostic and predictive biomarkers to aid in the selection of patients to receive the most-appropriate therapies - the biomarker field is one of the most challenging issues for oncologists today. Therefore, this has prompted us to commission a focus issue on biomarkers.

Identification of biomarkers and surrogate end points are important in clinical oncology. The review by Buyse and coauthors discusses the nature of prognostic and predictive biomarkers and surrogate end points, and examines the statistical techniques and clinical trial designs required for their validation. Surrogate end points are aimed at providing a faster or more-sensitive evaluation of the effect of a treatment and hence can substitute for a clinical end point. Importantly, surrogates require data to demonstrate that both the surrogate is prognostic of the true end point, and that the effect of treatment on the true end point correlates with the surrogate.

Even if a prognostic biomarker has a significant impact on the clinical outcome, it does not imply that the predictive accuracy of the biomarker is sufficient to justify its use in the clinic. However, the biological plausibility of a biomarker or surrogate might support its use even when full statistical validation is lacking. 'Selection' designs, in which only biomarker-positive patients are entered into a trial, are often adopted and although these trials can confirm the use of the biomarker for identifying those who will benefit from treatment, this is not a true test of prediction, because these studies provide no information on the lack of a benefit among marker-negative patients. In such situations, patients lacking the marker but who may respond to treatment could be missed, as was shown to be the case of anti-HER2 agents for breast cancer.

Gangadhar and Schilsky review the clinical development of molecular markers for individualizing therapy in colon cancer. They discuss the data showing that patients with stage II colon cancer and mismatch-repair-deficient tumors derive no benefit from 5-fluorouracil-based adjuvant therapy, whereas patients with stage III tumors do benefit from such treatment. Thus, microsatellite instability, a signature of deficient mismatch repair, is a strong predictive biomarker. Gangadhar and Schilsky recommend the testing for deficient mismatch repair in patients with sporadic stage II colon cancer who are considered for adjuvant chemotherapy.
What should physicians look for when evaluating prognostic gene-expression signatures? This question is addressed in an elegant review by Subramanian and Simon who focus on guidelines that physicians should refer to when evaluating studies on prognostic geneexpression signatures. They highlight that justification of validation studies for a new gene signature depends on the standard of care for the disease and the intended use of the signature. Interestingly, when they conducted a review of the literature of developmental or validation studies of prognostic signatures in non-small-cell lung cancer, they found that most studies failed to place adequate importance on patient selection or sample size planning. No prognostic signature for this disease has demonstrated sufficient utility to be incorporated into standard treatment guidelines.

There has long been controversy surrounding the use of CA125 testing for ovarian cancer. While this biomarker is useful for clinical monitoring of response to chemotherapy, it is far less robust as a tool for treatment initiation for recurrent disease. CA125 elevations often occur before any signs, symptoms or radiologic evidence of disease recurrence, prompting clinicians to consider this an early sign of relapse. Karam and Karlan review the evidence for treating asymptomatic patients with rising CA125 levels. They discuss the pivotal MRC OV05/ EORTC 55955 trial, in which patients with rising CA125 levels in complete remission after first-line chemotherapy were randomly assigned to either early intervention with therapy or had their therapy withheld until clinical evidence of recurrence. The overall survival was similar for both arms but, importantly, patients receiving early therapy had an impaired quality of life. Thus, routine CA125 testing might lead to patient anxiety, and withholding treatment in the event of rising CA125 might actually benefit patients.

These articles raise broader issues of how biomarker and surrogate end point adoption should be structured and standardized and the need for improved planning of developmental and validation studies of gene-signatures and biomarker studies. New biomarkers must be prospectively studied in large clinical trials and developmental studies conducted in parallel with phase I and II studies. Once validated, biomarkers should hold great promise to guide treatment selection for individualizing therapy for cancer patients.

doi:10.1038/nrclinonc.2010.70

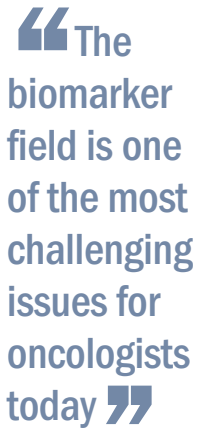

Lisa Hutchinson is the Editor of Nature Reviews Clinical Oncology. Vincent T. DeVita Jr is the Editor-in-Chief of Nature Reviews Clinical Oncology.

Competing interests The authors declare no competing interests. 